\title{
Histopathological evaluation of liver fibrosis and cirrhosis regression
}

\author{
Regina C. Lo', Haeryoung Kim² \\ 'Department of Pathology and State Key Laboratory for Liver Research, The University of Hong Kong, Hong Kong, China; ${ }^{2}$ Department \\ of Pathology, Seoul National University College of Medicine, Seoul National University Hospital, Seoul, Korea
}

The hepatic repair complex in the setting of cirrhosis has received increasing attention, as it implies the regression of cirrhosis, which was traditionally taken to be an irreversible state. In this brief review, the patterns of fibrosis, the existing staging systems for chronic liver disease and the histopathological features of cirrhosis regression are discussed. (Clin Mol Hepatol 2017;23:302-307)

Keywords: Liver fibrosis; Cirrhosis; Regression; Hepatic repair complex

\section{INTRODUCTION}

Fibrosis is an integral component of chronic liver disease. Ensuing inflammation of the liver due to various etiologies elicits the deposition of fibrous tissue in the parenchyma, which replaces the normal functional liver cells, remodels the vasculature in the organ, and compromises the liver function.' Liver fibrosis is well appreciated histologically on hematoxylin-eosin sections or with histochemical stains (Masson's trichrome or Sirius Red), which show collagen deposition with varying degrees of architectural distortion. Cirrhosis represents one end of the spectrum featuring diffuse fibrosis and formation of regenerative nodules. Advanced fibrosis of the liver has been traditionally taken as an irreversible state, and by itself is a risk factor for hepatocellular carcinoma. Therefore throughout the past decades the emphasis in the clinical management of chronic liver disease is to control inflammation and prevent fibrosis development or progression. This cornerstone concept has recently been revolutionized by the observation that regression of fibrosis and cirrhosis occurs.

\section{FIBROSIS PATTERN IN CHRONIC LIVER DISEASE}

The source of collagen in pathological conditions is believed to be the stellate cells. ${ }^{2}$ Upon chronic liver injury, hepatic stellate cells are activated and transformed into a myofibroblast-like phenotype to lay down extracellular matrix. ${ }^{3}$ Well-characterized stimuli to stellate cells include chronic inflammation, cytokine production by injured parenchymal cells, and disruption of extracellular matrix. Subsequent activation of hepatic stellate cells can be mediated through a number of signaling pathways. ${ }^{4}$

The patterns of liver fibrosis vary according to the etiology. In chronic hepatotropic virus infection, portal expansion is followed by periportal fibrosis, septal (bridging) fibrosis, and cirrhosis. For

\section{Corresponding author: Haeryoung Kim}

Department of Pathology, Seoul National University College of Medicine, Seoul National University Hospital, 103 Daehak-ro, Jongno-gu, Seoul 03080, Korea

Tel: +82-2-740-8322, Fax: +82-2-765-5600

E-mail: haeryoung.kim@snu.ac.kr

http://orcid.org/0000-0002-4205-9081

Received : Dec. 5, 2017 / Accepted : Dec. 11, 2017

Copyright $\odot 2017$ by The Korean Association for the Study of the Liver

This is an Open Access article distributed under the terms of the Creative Commons Attribution Non-Commercial License (http://creativecommons.org/licenses/by-nc/3.0/) which permits unrestricted non-commercial use, distribution, and reproduction in any medium, provided the original work is properly cited. 
alcoholic liver disease or adult non-alcoholic fatty liver disease, fibrosis starts with a centrilobular perivenular distribution and sinusoidal fibrosis. ${ }^{5,6}$ The fibrosis pattern in pediatric fatty liver disease is similar to that observed in hepatotropic virus infection featuring a periportal distribution, while perisinusoidal or perivenular fibrosis is usually not apparent. Cirrhosis resulting from biliary tract disease is characterized by irregular-shaped nodules ("jigsaw" micronodular pattern) and the presence of prominent "halos", due to feathery degeneration of periseptal hepatocytes. In venous outflow obstruction, fibrosis progressively links the adjacent central veins and portal tracts, resulting in veno-centric ("reversed lobulation" cirrhosis) or veno-portal cirrhosis.

\section{STAGING SYSTEMS FOR LIVER FIBROSIS}

The first attempt at a semi-quantitative scoring system for chronic hepatitis was proposed by Knodell, et al. in the early 1980 's. ' However, scores for stage and grade were combined into a single numerical value in this system. Since then, other methods scoring grade and stage separately have been proposed, including the Scheuer system, ${ }^{8}$ the Batts-Ludwig system, ${ }^{9}$ Ishak system ${ }^{10}$ and METAVIR system." A grading and staging system was also proposed by the Korean Study Group for the Pathology of Digestive Diseases. ${ }^{12}$ Although there are minor differences between the different systems for fibrosis stage, the basic concept is similar: fibrosis begins in the portal tracts, extends to adjacent portal tracts and terminal hepatic venules resulting in architectural distortion and ultimately to cirrhosis.

Adaptations of these staging systems have been proposed for other chronic liver diseases where the fibrosis patterns differ from the portal-based chronic viral hepatitis. For example the Non-alcoholic Steatohepatitis Clinical Research Network (NASH CRN) histologic scoring system includes stages $1 \mathrm{~A}$ and $1 \mathrm{~B}$ for zone $3 \mathrm{fi}$ brosis while also providing a stage $1 \mathrm{C}$ for pediatric-type fibrosis. ${ }^{13}$ The Congestive Hepatic Fibrosis score is another example where stage 1 refers to zone 3 fibrosis. ${ }^{14}$

Whatever staging schema is used for chronic viral hepatitis, fatty
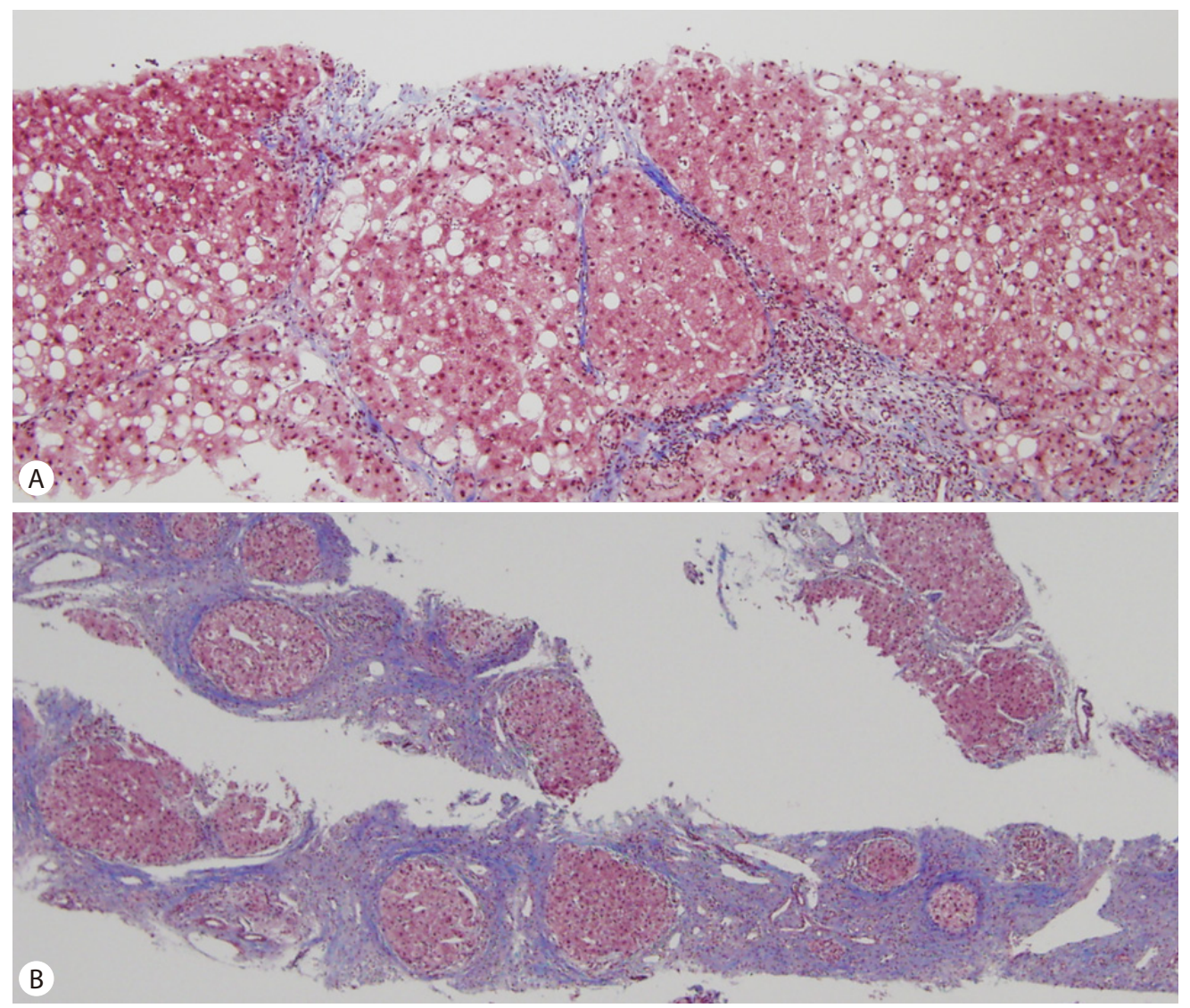

Figure 1. (A) Mixtures of thin and broad fibrous septa are seen in this case of cirrhosis with Laennec stage 4B. (B) A needle biopsy of Laennec stage 4C cirrhosis demonstrating very broad fibrous septa with micronodules (Masson's trichrome stain, original magnification $\times 100[\mathrm{~A}], \times 40[\mathrm{~B}]$ ). 
liver disease, biliary cholangitis and congestive hepatic fibrosis, the final score "stage 4" refers to cirrhosis for all systems. The Laennec system further subclassifies the degree of cirrhosis by subdividing METAVIR stage 4 into stages $4 A, 4 B$ and $4 C$, based on the thickness of fibrous septa and size of the nodules on liver biopsies. ${ }^{15}$ Stage $4 \mathrm{~A}$ is characterized by mild cirrhosis (definite or probable), where most septa are thin. Stage $4 B$ is moderate cirrhosis showing at least two broad fibrous septa without very broad septa, while Stage $4 \mathrm{C}$ refers to severe cirrhosis in which at least one very broad septum or many micronodules are present (Fig. 1). This staging system was demonstrated to be well-correlated with the clinical stage of cirrhosis, degree of portal hypertension and risk of recurrence of hepatocellular carcinoma after curative resection. ${ }^{16,17}$

However, all of the aforementioned staging systems have been developed based on the histological findings of previously untreated chronic liver diseases, and the concept of cirrhosis regression was not taken into account.

\section{CLINICOPATHOLOGICAL EVIDENCE OF CIR- RHOSIS REGRESSION}

The notion of regressing cirrhosis in human livers was first proposed by Wanless, et al. in year 2000, in a clinicopathological analysis of 52 explanted livers with cirrhosis or incomplete septal cirrhosis of various etiologies including hepatitis B, hepatitis C, alcoholism and primary biliary cirrhosis. ${ }^{18}$ They described in detail the histological features associated with cirrhosis regression, collectively known as "hepatic repair complex": perforated delicate septa, isolated thick collagen fibers (not visibly attached to portal tracts, venules or septa), delicate periportal fibrous spikes, hepatic vein remnants with prolapsed hepatocytes (hepatocytes within lumens of hepatic veins), hepatocytes within portal tracts or split septa (clusters or cords of hepatocytes identified within portal tracts or trapped in fibrous septa), minute regenerative nodules, and aberrant parenchymal veins (within 5-hepatocyte diameter from portal tracts) (Fig. 2). ${ }^{18}$ These features probably represent a reparative process and dissolution of formed fibrosis. Subsequent to the first report by Wanless, et al, clinical studies have demonstrated the regression of fibrosis in chronic liver disease, including chronic viral hepatitis after anti-viral treatment, non-alcoholic fatty liver disease after bariatric surgery, and in cardiac cirrhosis after heart transplantation. In these studies, degree of fibrosis was mostly assessed by transient elastography and/or histology. ${ }^{19-24}$

Major theories accounting for regression of liver fibrosis/cirrhosis include cessation of chronic injury to the liver, inactivation of transformed hepatic stellate cells, degradation of extracellular matrix, and shifting of microenvironment to a restoration type. These postulations are in line with our current understanding on liver fibrogenesis. Based on these fibrosis regression theories, the molecular mechanisms underlying the process have been delineated and therapeutic opportunities have been opened up. ${ }^{3,25-27}$

\section{HISTOLOGICAL ASSESSMENT OF FIBROSIS/ CIRRHOSIS REGRESSION}

In practice, regression of fibrosis is defined as the decrease in
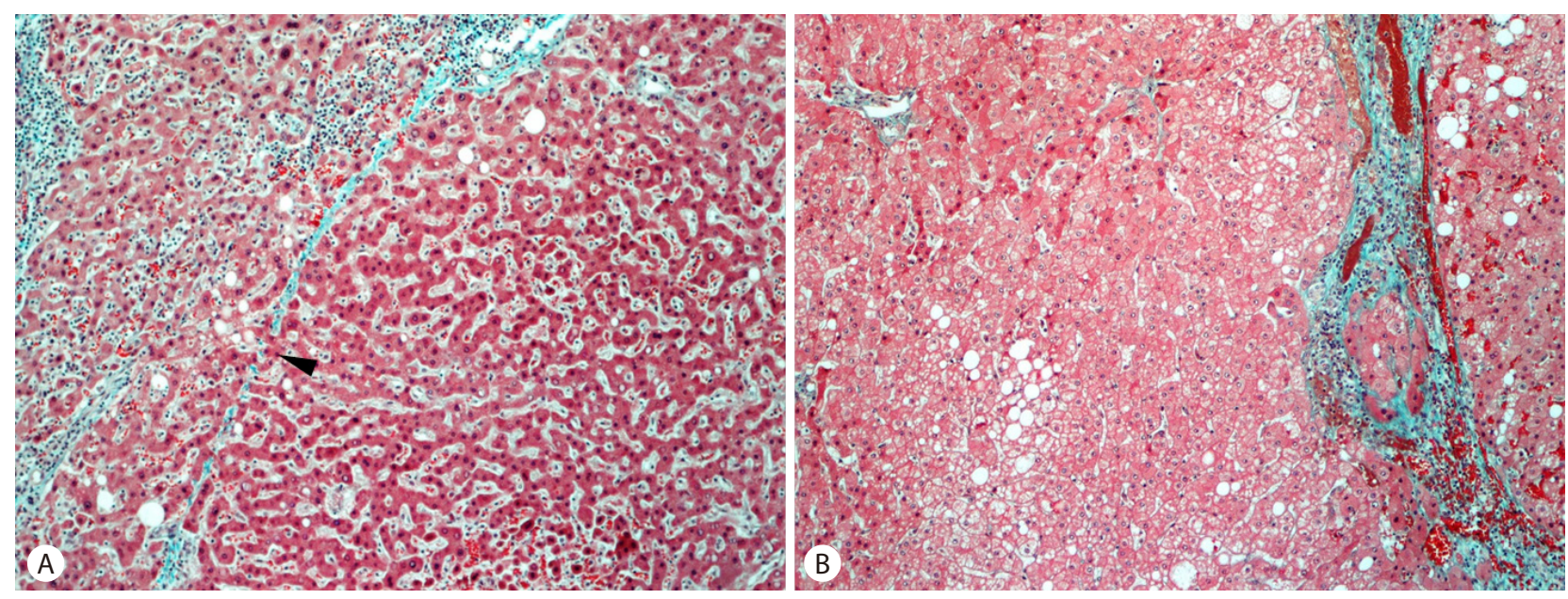

Figure 2. Histological features of fibrosis regression. (A) Presence of delicate perforated fibrous septa (arrowhead) and (B) clusters of hepatocytes within split septa (Masson's trichrome stain, original magnification $\times 100$ ). 

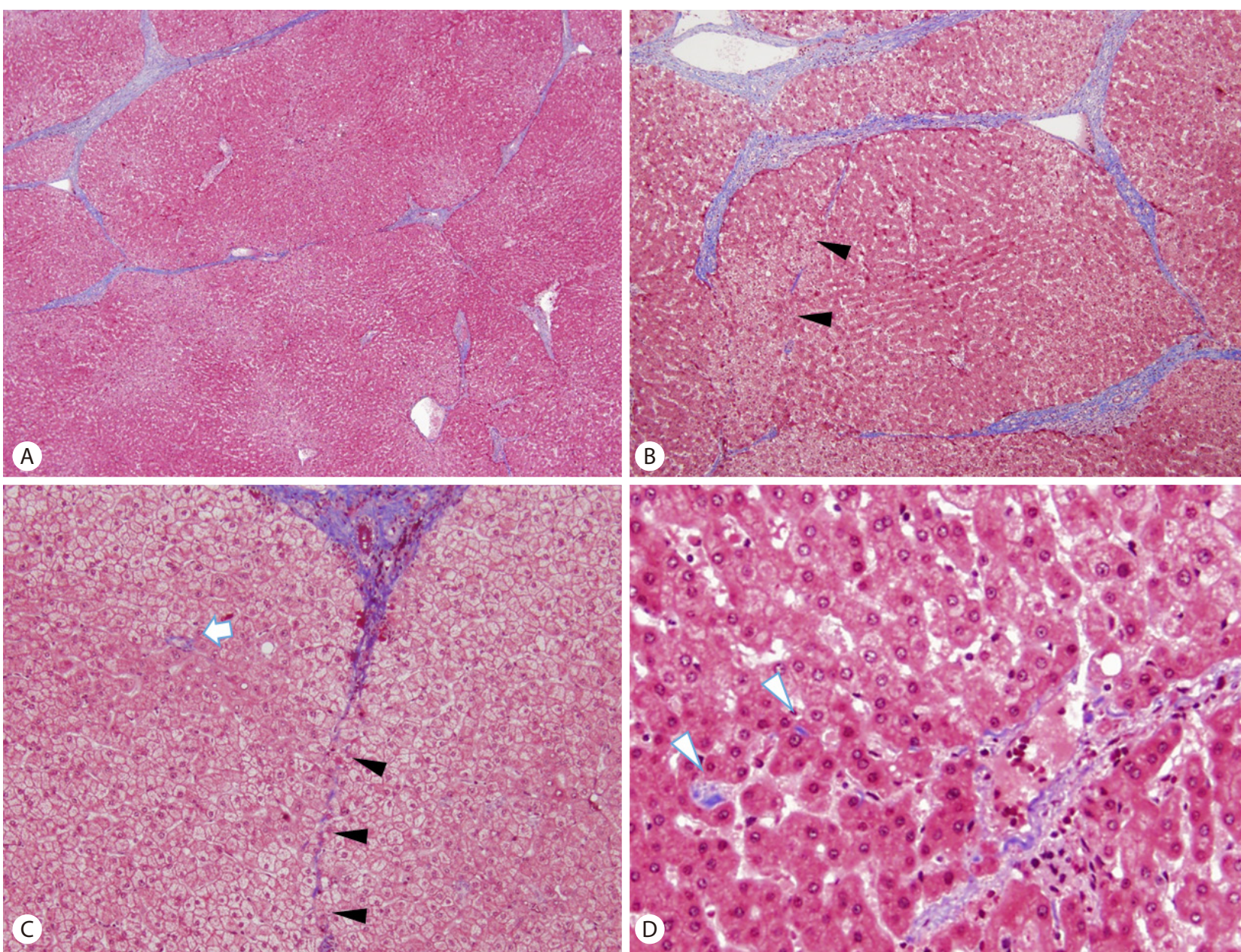

Figure 3. An example of a cirrhosis with predominantly regressive pattern. (A) Cirrhosis with thin fibrous septa, corresponding to Laennec stage 4A. (B, $C, D)$ Features of cirrhosis regression are seen in the field adjacent to (A), including perforated delicate septa (B, C; arrowheads), remnant portal tracts (C; arrow) and isolated collagen fibers (D; arrowheads). (Masson's trichrome stain, original magnification $\times 40$ (A, B), ×100 (C), ×200 (D))

fibrosis score in paired consecutive biopsies, whichever scoring system is used. The differences in fibrosis scores have served as histological outcomes in clinical trials evaluating the effect of various new drugs. However, the limitations of paired biopsy evaluation include the critical issue of sample size, as the length of the liver biopsy and the number of portal tracts included (ideally $2 \mathrm{~cm}$ or longer, and containing 11 or more complete portal tracts) may influence the fibrosis score. ${ }^{28}$ In this regard, the hepatic repair complex may be a useful feature for assessment of fibrosis regression in cases with histologically proven cirrhosis, as the presence of the pertinent histological findings implies regression of cirrhosis.

Recently, Sun, et al. proposed the "Beijing classification" which evaluates the quality of fibrosis in individuals with chronic hepatitis B, pre- and post-entecavir-based therapy. ${ }^{29}$ Fibrosis was classified as "predominantly progressive", "indeterminate" and "predominantly regressive" based on low-power examination of hematoxylin-eosin, trichrome and reticulin-stained sections as follows: 1) "Predominantly progressive": most fibrous septa were broad, with loosely aggregated collagen fibers that were pale staining on trichrome stain, and contained inflammatory cells and ductular reactions; 2) "Indeterminate": a balance between progressive and regressive fibrosis; and 3) "Predominantly regressive": most fibrous septa showed features of the hepatic repair complex, with thin, dense and acellular stroma staining dark blue on trichrome stain (Fig. 3). By this method, chronic hepatitis B was classified into cases showing regression of fibrosis and those at risk for continued progression despite antiviral treatment. When used together with existing methods of grading and staging, this method may add another dimension to the current crosssectional evaluation of grading and staging: a dynamic evaluation of the direction of fibrosis (progression versus regression). ${ }^{29,30} \mathrm{Al}$ though further validation is required, this suggests another role 
for the liver biopsy in the evaluation of chronic liver disease.

The histological features of fibrosis regression are less easy to find on biopsied liver specimens compared to resected tissues. In addition, as the hepatic repair complex has been proposed as a feature of cirrhosis regression, it implies the presence of an already established cirrhosis. This could pose difficulties when interpreting liver biopsies where no definite cirrhotic nodules are seen and only thin fibrous septa are present; indeed, cases signed out as incomplete septal fibrosis could actually be regressed cirrhosis. The identification of some of the aforementioned features of the hepatic repair complex, and thus signs of parenchymal remodeling, could help make the diagnosis of regression of cirrhosis.

\section{IMPLICATIONS, UNANSWERED QUESTIONS AND FUTURE PERSPECTIVES}

As cirrhosis is a heterogeneous condition with regard to scarring pattern, rates of progression, and the potential for regression, it has been suggested that the term "cirrhosis" be replaced by "advanced stage" fibrosis when diagnosing chronic liver diseases, together with information including etiology, activity grade and features of progression or regression. ${ }^{31}$

While regression of fibrosis appears encouraging news, certain issues remain to be clarified. Regarding the methods of fibrosis assessment, sampling error for liver biopsy is a known issue and assessment should be based on adequate specimens. ${ }^{32,33}$ Besides, discrepancy between morphometric analysis and liver stiffness measurement was observed in some scenarios. ${ }^{34}$ This implies simultaneous use of multiple methods to validate the degree of liver fibrosis may be indicated in order to obtain a better picture of the fibrosis content in tissue.

With further understanding on regression of fibrosis/cirrhosis, the clinical course and treatment plans for chronic liver disease will be reformed. Indication and treatment regimens for chronic hepatitis may need to be revised and more resources on monitoring treatment response in terms of fibrosis will need to be implemented in the health care system. This may also imply a decrease in the risk of hepatocellular carcinoma development in chronic liver disease. Moreover, while existing evidences mainly focus on the removal of the triggering liver insults to promote fibrosis regression (for example, anti-virals for chronic hepatitis, bariatric surgery for non-alcoholic liver disease, venesection for hemochromatosis, and copper chelation for Wilson disease, etc), there is still room for investigation on the role of adjuvant anti-fibrotic therapies in promoting fibrosis regression. In this regard, a recent study on LOXL2 mAb is a good example to understand how dissection of the molecular basis of fibrogenesis may provide treatment options for anti-fibrotic therapies. ${ }^{35}$ Given that liver fibrosis/cirrhosis is a heterogeneous disease as reflected by clinical behaviors and histological clues, identification and validation of clinicopathological parameters to predict the likelihood of fibrosis regression in specific patients would warrant further investigation. As far as histological assessment is concerned, future efforts to incorporate features of regression in the staging system of chronic liver diseases are awaited.

\section{Authors' contribution}

Lo RC, Kim $\mathrm{H}$ have both contributed to the design, writing and final approval of this review.

\section{Financial support}

This work was supported by the Basic Science Research Program through NRF funded by the Ministry of Education (NRF2016R1D1A1A09919042).

\section{Conflicts of Interest}

The authors have no conflicts to disclose.

\section{REFERENCES}

1. Rappaport AM, MacPhee PJ, Fisher MM, Phillips MJ. The scarring of the liver acini (Cirrhosis). Tridimensional and microcirculatory considerations. Virchows Arch A Pathol Anat Histopathol 1983:402:107-137.

2. Bouwens $L$, Baekeland $M$, Wisse E. Cytokinetic analysis of the expanding Kupffer-cell population in rat liver. Cell Tissue Kinet 1986;19:217-226.

3. Friedman SL. Hepatic stellate cells: protean, multifunctional, and enigmatic cells of the liver. Physiol Rev 2008;88:125-172.

4. Tsuchida T, Friedman SL. Mechanisms of hepatic stellate cell activation. Nat Rev Gastroenterol Hepatol 2017;14:397-411.

5. Brunt EM, Janney CG, Di Bisceglie AM, Neuschwander-Tetri BA, Bacon BR. Nonalcoholic steatohepatitis: a proposal for grading and staging the histological lesions. Am J Gastroenterol 1999;94:2467-2474.

6. Van Waes L, Lieber CS. Early perivenular sclerosis in alcoholic fatty liver: an index of progressive liver injury. Gastroenterology 1977;73:646-650.

7. Knodell RG, Ishak KG, Black WC, Chen TS, Craig R, Kaplowitz N, et al. Formulation and application of a numerical scoring system for assessing histological activity in asymptomatic chronic active hepa- 
titis. Hepatology 1981;1:431-435.

8. Scheuer PJ. Classification of chronic viral hepatitis: a need for reassessment. J Hepatol 1991;13:372-374.

9. Batts KP, Ludwig J. Chronic hepatitis. An update on terminology and reporting. Am J Surg Pathol 1995;19:1409-1417.

10. Ishak K, Baptista A, Bianchi L, Callea F, De Groote J, Gudat F, et al. Histological grading and staging of chronic hepatitis. J Hepatol 1995;22:696-699.

11. Bedossa P, Poynard T. An algorithm for the grading of activity in chronic hepatitis C. The METAVIR Cooperative Study Group. Hepatology 1996;24:289-293.

12. Park YN, Kim HG, Chon CY, Park JB, Sohn JH, Yang SH, et al. Histological grading and staging of chronic hepatitis: standardized guideline proposed by the Korean Study Group for the Pathology of Digestive Diseases. Korean J Pathol 1999;33:337-334.

13. Kleiner DE, Brunt EM, Van Natta M, Behling C, Contos MJ, Cummings $\mathrm{OW}$, et al. Design and validation of a histological scoring system for nonalcoholic fatty liver disease. Hepatology 2005;41:1313-1321.

14. Dai DF, Swanson PE, Krieger EV, Liou IW, Carithers RL, Yeh MM. Congestive hepatic fibrosis score: a novel histologic assessment of clinical severity. Mod Pathol 2014;27:1552-1558.

15. Wanless IR, Sweeney G, Dhillon AP, Guido M, Piga A, Galanello R, et al. Lack of progressive hepatic fibrosis during long-term therapy with deferiprone in subjects with transfusion-dependent betathalassemia. Blood 2002;100:1566-1569.

16. Kim MY, Cho MY, Baik SK, Park HJ, Jeon HK, Im CK, et al. Histological subclassification of cirrhosis using the Laennec fibrosis scoring system correlates with clinical stage and grade of portal hypertension. J Hepatol 2011;55:1004-1009.

17. Kim SU, Oh HJ, Wanless IR, Lee S, Han KH, Park YN. The Laennec staging system for histological sub-classification of cirrhosis is useful for stratification of prognosis in patients with liver cirrhosis. J Hepatol 2012;57:556-563.

18. Wanless IR, Nakashima E, Sherman M. Regression of human cirrhosis. Morphologic features and the genesis of incomplete septal cirrhosis. Arch Pathol Lab Med 2000;124:1599-1607.

19. Dienstag JL, Goldin RD, Heathcote EJ, Hann HW, Woessner M, Stephenson SL, et al. Histological outcome during long-term lamivudine therapy. Gastroenterology 2003;124:105-117.

20. Marcellin P, Gane E, Buti M, Afdhal N, Sievert W, Jacobson IM, et al. Regression of cirrhosis during treatment with tenofovir disoproxil fumarate for chronic hepatitis B: a 5-year open-label follow-up study.
Lancet 2013;381:468-475.

21. Chang TT, Liaw YF, Wu SS, Schiff E, Han KH, Lai CL, et al. Long-term entecavir therapy results in the reversal of fibrosis/cirrhosis and continued histological improvement in patients with chronic hepatitis B. Hepatology 2010;52:886-893.

22. Ellis EL, Mann DA. Clinical evidence for the regression of liver fibrosis. J Hepatol 2012;56:1171-1180.

23. Crespo-Leiro MG, Robles O, Paniagua MJ, Marzoa R, Naya C, Flores $X$, et al. Reversal of cardiac cirrhosis following orthotopic heart transplantation. Am J Transplant 2008;8:1336-1339.

24. Lassailly G, Caiazzo R, Buob D, Pigeyre M, Verkindt H, Labreuche J, et al. Bariatric Surgery Reduces Features of Nonalcoholic Steatohepatitis in Morbidly Obese Patients. Gastroenterology 2015;149:379-388.

25. Iredale JP. Hepatic stellate cell behavior during resolution of liver injury. Semin Liver Dis 2001;21:427-436.

26. Uchinami H, Seki E, Brenner DA, D'Armiento J. Loss of MMP 13 attenuates murine hepatic injury and fibrosis during cholestasis. Hepatology 2006;44:420-429.

27. Tacke F, Trautwein C. Mechanisms of liver fibrosis resolution. J Hepatol 2015;63:1038-1039.

28. Rockey DC, Caldwell SH, Goodman ZD, Nelson RC, Smith AD. Liver biopsy. Hepatology 2009;49:1017-1044.

29. Sun Y, Zhou J, Wang L, Wu X, Chen Y, Piao H, et al. New classification of liver biopsy assessment for fibrosis in chronic hepatitis $B$ patients before and after treatment. Hepatology 2017;65:1438-1450.

30. Kleiner DE. On beyond staging and grading: Liver biopsy evaluation in a posttreatment world. Hepatology 2017;65:1432-1434.

31. Hytiroglou P, Snover DC, Alves V, Balabaud C, Bhathal PS, BioulacSage $P$, et al. Beyond "cirrhosis": a proposal from the International Liver Pathology Study Group. Am J Clin Pathol 2012;137:5-9.

32. Colloredo G, Guido M, Sonzogni A, Leandro G. Impact of liver biopsy size on histological evaluation of chronic viral hepatitis: the smaller the sample, the milder the disease. J Hepatol 2003;39:239-244.

33. Scheuer PJ. Liver biopsy size matters in chronic hepatitis: bigger is better. Hepatology 2003;38:1356-1358.

34. Wong GL, Wong VW, Choi PC, Chan AW, Chum RH, Chan HK, et al. Assessment of fibrosis by transient elastography compared with liver biopsy and morphometry in chronic liver diseases. Clin Gastroenterol Hepatol 2008;6:1027-1035.

35. Ikenaga N, Peng ZW, Vaid KA, Liu SB, Yoshida S, Sverdlov DY, et al. Selective targeting of lysyl oxidase-like 2 (LOXL2) suppresses hepatic fibrosis progression and accelerates its reversal. Gut 2017;66:1697-1708. 